\title{
A Case Of Paraneoplasic Limbic Encephalitis During The Course Of Depression
}

\section{${ }^{1}$ Mohamed Habib Aoun, ${ }^{1}$ Salma Sakka, ${ }^{1}$ Nouha Farhat, ${ }^{1}$ Hanene Hadj Kacem, ${ }^{1}$ Olfa Hdiji, ${ }^{1}$ Mariem Damak, ${ }^{1}$ Chokri Mhiri. \\ ${ }^{1}$ Neurology Department, Habib Bourguiba Hospital, Tunisia}

\section{Background and Aims:}

Limbic encephalitis is characterized by irritability, depression, sleeping disorders, seizure, hallucination and short-term memory loss.

Most of the patients evolve with confusion and epileptic temporal lobe seizures.

Previously associated with cancer, recent evidence suggests that limbic encephalitis in fact more often occurs independently of cancer, as an autoimmune phenomenon.

Here we describe a case with a two-week history of psychiatric impairment, who was later diagnosed with Limbic Encephalitis.

\section{Case Report:}

This is a 32-year-old female patient, with a history of Thyroid Papillary carcinoma. She had a total thyroidectomy in 2015 with lodotherapy. She had been taking since Levothyroxin.

She presented apathy, anorexia and adynamia with memory loss. Two weeks later, she presented an epileptic seizure. By this time, she was started on carbamazepine $1 \mathrm{mg}$ once daily.

Laboratory assessment was performed and revealed normal urinalysis complete blood count, basic metabolic panel, hepatic function panel, thyroid-stimulating hormone, free thyroxine, ammonia, folate, and vitamin B12. HIV testing and urine toxicology screen were negative.

Cerebrospinal fluid (CSF) was colorless, with only one white blood cell (monocyte), one erythrocyte, glucose level of $65 \mathrm{mg} / \mathrm{dL}$ (normal range, 40 $85 \mathrm{mg} / \mathrm{dL}$ ), and protein level of $43 \mathrm{mg} / \mathrm{dL}$ (normal range, $15-45 \mathrm{mg} / \mathrm{dL}$ ). CSF was negative for herpes simplex virus via PCR. CSF cytology was negative, and CSF protein electrophoresis did not identify any oligoclonal bands.

MRI of the brain, with and without contrast, showed hyperintense signals from both hippocampi, highly suggestive of limbic encephalitis presenting as a paraneoplastic manifestation of papillary carcinoma.

EEG showed a disorganized background with bitemporal slowing consistent with encephalopathy.

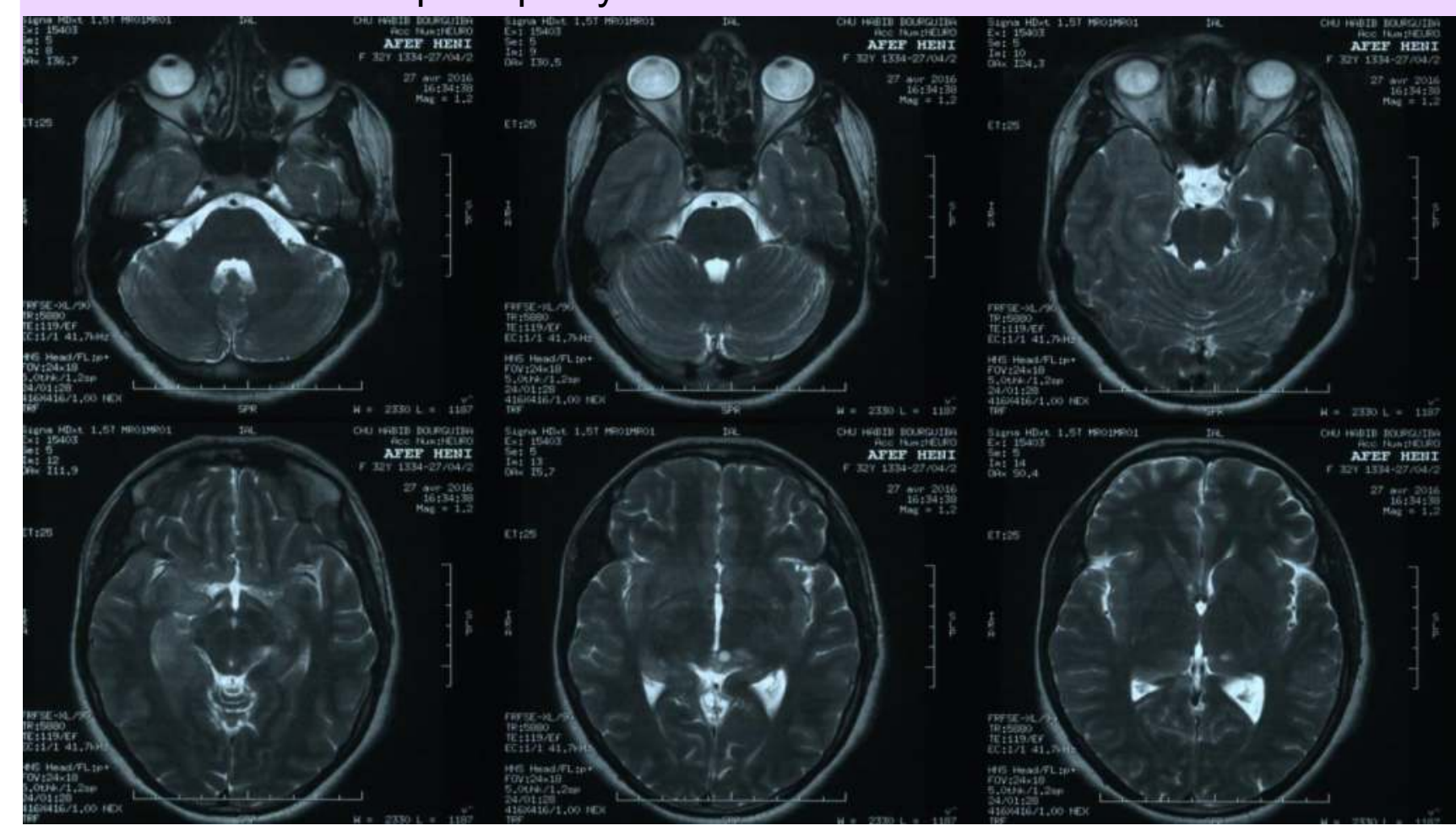

Fig 1. Hypersignal $\mathrm{T} 2$ in temporal lobes, predominant on the right side, extended to the hippocampal and thalamic region without contrast enhancement

\section{Discussion:}

Limbic encephalitis is a paraneoplastic neurological disorder in which, typically, the neurological symptoms occur before the cancer is diagnosed [1]. In our case, the patient experienced neuropsychiatric symptoms after the cancer treatment. its symptoms are similar to those reported by the literature.

Neuropsychiatric symptoms include short-term-memory disturbance epileptic seizures, confusion of acute onset, changes in personality hallucinations, depression and cognitive disturbances [1]

Although less frequent than depression, anxiety is also observed with focal brain lesions, usually involving the temporolimbic lobe [2] Considering the importance of the hippocampus in both emotional regulation and memory, one would not be surprised to learn that hippocampal activation patterns of humans differ according to attachment status.[3]

Benke et al. also reported a case with limbic and cerebellar paraneoplastic syndrome which was associated with a squamous lung carcinoma, who had severe anterograde memory loss, frontal executive dysfunction and behavioural alterations. Brain MRI of this case revealed inflammatory changes followed by progressive atrophy affecting the cerebellum and both temporal lobes [4].

Because the depressive symptoms of our patient were acute, of lateonset and she had no pre-morbid symptoms, an organic etiology was primarily suspected. According to the diagnostic criteriaused by Gultekin et al., MRI findings,clinical symptoms, neuropsychological and labora-tory findings and exclusion of other aetiologies for encephalitis (i.e. herpes virus, neurosyphilis), the case was diagnosed as limbic encephalitis.

\section{Conclusion:}

Paraneoplastic neurological syndromes are rare occurring in less than $1 \%$ of patients with malignancies. The most frequent neoplasms associated with paraneoplastic limbic encephalitis are small cell lung cancer, testicular tumors, thymoma, Hodgkin's lymphoma and breast cancer. It is important to consider paraneoplastic syndromes such as limbic encephalitis early on when incongruous neuropsychiatric symptoms are present, as early diagnosis of malignancy and prompt initiation of treatment can lead to improvement in the neuropsychiatric manifestations. 